\title{
Warnsignale in der Parodontologie - Was der Kieferorthopäde wissen sollte
}

\section{Periodontal Warning Signals - What the Orthodontist Should Know}

\author{
Autoren \\ J. P. Woelber, P. Ratka-Krüger \\ Institut \\ Klinik für Zahnerhaltungskunde und Parodontologie, Department für Zahn-, Mund- und Kieferheilkunde, \\ Universitätsklinikum Freiburg
}

\author{
Schlüsselwörter \\ - Parodontitis \\ - Risikofaktor \\ - kieferorthopädische \\ Therapie \\ Key words \\ - periodontitis \\ - risk factor \\ orthodontic therapy
}

Bibliografie

DOI http://dx.doi.org/

10.1055/s-0042-111899

Inf Orthod Kieferorthop 2016;

48: 171-175

(c) Georg Thieme Verlag KG

Stuttgart · New York

ISSN 0020-0336

Korrespondenzadresse

\section{Dr. Johan Wölber}

Klinik für Zahnerhaltungskunde und Parodontologie

Department für Zahn-, Mundund Kieferheilkunde Universitätsklinikum Freiburg Hugstetter Straße 55 79106 Freiburg

Tel.: $+49 / 761 / 27048800$

Fax: $+49 / 761 / 27047620$

johan.woelber@uniklinik-

freiburg.de

\section{Zusammenfassung \\ $\nabla$}

Kieferorthopädische Therapien ergänzen oft den Abschluss einer parodontalen Therapie, sie bergen jedoch manchmal auch ein Risiko für die Entstehung von parodontalen Pathologien, wie etwa Rezessionen, Gingivitis oder die Progression einer Parodontitis. Nicht selten stellt sich auch ein Patient mit einer unerkannten, nicht diagnostizierten Parodontitis wegen Stellungsänderungen der Zähne als erstes beim Kieferorthopäden vor. Die vorliegende Literaturübersicht befasst sich daher mit parodontalen Warnsignalen bzw. Frühsymptomen, die in der kieferorthopädischen Therapie berücksichtigt werden sollten. Für ein besseres Verständnis werden die aktuellen ätiologischen Theorien zur Entstehung der Gingivitis und Parodontitis, die notwendige parodontale Diagnostik und derzeitige Therapiemethoden beleuchtet. Zusammenfassend lässt sich sagen, dass durch Erkennen von parodontalen Risikofaktoren, klinischer Inspektion und parodontaler Sondierung die Entstehung von parodontalen Erkrankungen frühzeitig erkannt oder durch Einleitung von präventiven Maßnahmen (wie regelmäßiger Motivation und Instruktion zur Mundhygiene, professionelle Zahnreinigungen, Zusammenarbeit mit dem Parodontologen) verhindert werden können.

\section{Einführung und ätiologische Aspekte der Parodontitis \\ $\nabla$}

Die chronische Parodontitis wird durch die Deutsche Gesellschaft für Parodontologie definiert als „Infektionserkrankung, die zur Entzündung des Zahnhalteapparates sowie zu progressiven Attachment- und Knochenverlust führt.“ [1]. Einen Meilenstein in der Ätiologie der parodontalen Erkrankungen stellten die Untersuchungen zur experimentellen Gingivitis von Löe, Theilade u. Jensen [2] dar, die eine starke positive Korrela-

\section{Abstract \\ $\nabla$}

Orthodontic therapies often complement periodontal therapies, but sometimes they harbor the risk of the development of periodontal pathologies, like gingival recessions, gingivitis or the progression of periodontal disease. Occasionally, patients with undiagnosed, unknown periodontal disease are primarily consulting their orthodontist due to malposition of their teeth. This review wants to focus on periodontal warning signals and early signs of periodontal diseases, which are necessary to be checked before and during orthodontic therapy. For a better understanding, current etiologic theories about the development of gingivitis and periodontal disease, necessary diagnostic procedures and current treatment strategies are going to be presented. To summarize, it can be stated that due to early identification of risk factors and preventive treatment (like regular motivation and instruction for adequate oral hygiene, professional tooth cleanings, and collaboration with the periodontist) the development of periodontal pathologies can be prevented. tion zwischen dem Vorhandensein von dentaler Plaque und gingivaler Entzündung feststellen konnten. Durch diese Feststellung beruhen die heutigen therapeutischen Konzepte im Wesentlichen auf einer Kontrolle des oralen Biofilms. Es zeigte sich allerdings im Laufe der Zeit, dass nicht jede Art von Plaque eine gleich starke entzündliche Antwort provozierte, sondern dass sowohl die Art der bakteriellen Komposition als auch die immunologischen Reaktionen des Wirtsorganismus eine entscheidende Rolle spielen [3-5]. Die heutigen ätiologischen Theorien gehen davon 
aus, dass der Wirtsorganismus den parodontalpathogenen Erregern eine entzündliche Umgebung bieten muss, um eine Verschiebung zwischen gesundheitsförderlichen und parodontalpathogenen Keimen zugunsten der krankheitsfördernden Keime (Dysbiose) zu ermöglichen und zu unterhalten [5,6]. Im Wesentlichen stehen hierbei die Faktoren des erworbenen und angeborenen Immunsystems [7] sowie pro-inflammatorische Faktoren und der oxidative Stress im Vordergrund $[8,9]$. Die häufigsten Formen der parodontalen Erkrankungen stellen die chronische Parodontitis und mit geringeren Prävalenzen die aggressive Parodontitis dar, bei denen aus ätiologischer Sicht der aggressiven Parodontitis eine stärke genetische Disposition zugerechnet wird $[10,11]$. Aus diesem Grund sollte anamnestisch auch immer nach familiären Häufungen von Parodontalerkrankungen gefragt werden.

\section{Checkliste der parodontologischen Warnhinweise:}

- positive Familienanamnese bezüglich parodontaler Erkrankungen

- PSI $^{*}>$ Grad 3 und 4

- Risikofaktoren wie Alter, Rauchen, Diabetes mellitus, Metabolisches Syndrom

- Syndrome wie Down-Syndrom, Leukozyten-Adhäsionsdefekt-Syndrom, Chediak-Higashi-Syndrom, PapillonLefévre-Syndrom

- Rezessionen

$\checkmark$ freiliegende Furkationen

${ }^{*}$ PSI $=$ Parodontaler Screening Index

\section{Prävalenz der Parodontalerkrankungen}

$\nabla$

Epidemiologische Studien schätzen, dass weltweit 743 Millionen Menschen von Parodontitis betroffen sind. Nach den Daten der letzten deutschen Mundgesundheitsstudie (DMS IV) kann man davon ausgehen, dass in Deutschland 53\% der Erwachsenen an einer mittelschweren und $20 \%$ an einer schweren Parodontitis erkrankt sind, mit nochmals häufigerem Erkrankungsauftreten im hohen Alter [12]. Die Prävalenzen waren damit im Vergleich zur vorhergehenden Mundgesundheitsstudie um mehr als 20\% zunehmend. Bezüglich der aggressiven Parodontitis schwanken die Prävalenzen zwischen $0,1-15 \%$ je nach geografischen und ethnischen Faktoren, mit höheren Prävalenzen bei Afro-Amerikanern und geringen Prävalenzen bei Kaukasiern $[13,14]$.

\section{Risikofaktoren der Parodontitis}

$\nabla$

Basierend auf den ätiologischen Modellen der Parodontitis existieren eine Vielzahl von Risikofaktoren, welche sowohl die Art und Menge der dentalen Plaque als auch die immunologische Antwort des Wirtsorganismus modifizieren. Zu den wichtigsten Risikofaktoren gehören das Alter des Patienten, Rauchen, Diabetes mellitus, Stress, Übergewicht und genetische Faktoren $[10,15,16]$. Des Weiteren können Zahnfehlstellungen, überhängende Restaurationen, Zahnanomalien und Furkationsbeteiligungen als Plaqueretentionstellen eine Progression der Parodontitis lokal begünstigen. Zudem gehen bestimmte Syndrome wie Down-Syndrom, Leukozyten-Adhäsionsdefekt-Syndrom, Chediak-Higashi-Syndrom oder Papillon-Lefévre-Syndrom mit schweren parodontalen Defekten einher, die auch schon das Milchgebiss betreffen können. Beim Vorhandensein der genannten Risikofaktoren sollte eine weitergehende parodontale Untersuchung eingeleitet werden.

\section{Diagnostik in der Parodontologie}

$\nabla$

Nicht selten konsultieren Patienten bei unerkannter Parodontitis wegen Zahnstellungsanomalien (Lücken, Elongationen) zunächst ihren Kieferorthopäden ( $\bullet$ Abb. 1, 2). Deshalb sollte dieser zunächst immer eine Basisdiagnostik des Parodonts vornehmen. Als wichtigstes Instrument zur Basisdiagnostik von Parodontalerkrankungen dient der Parodontale Screening Index (PSI) $[17,18]$, der mit einer WHO-Sonde erhoben wird ( $\bullet$ Abb. 3). Für die Beurteilung wird das Gebiss in Sextanten unterteilt und der ermittelte Wert in 4 verschiedenen Codes ausgedrückt ( $\bullet$ Tab. 1). Bei Kindern und Jugendlichen werden die Werte nur an den Zähnen 16, 11, 26, 36, 31 und 46 erhoben. Vor Beginn einer kieferorthopädischen Therapie sollte der PSI durch den Kieferorthopäden oder den behandelnden Hauszahnarzt durchgeführt worden sein.

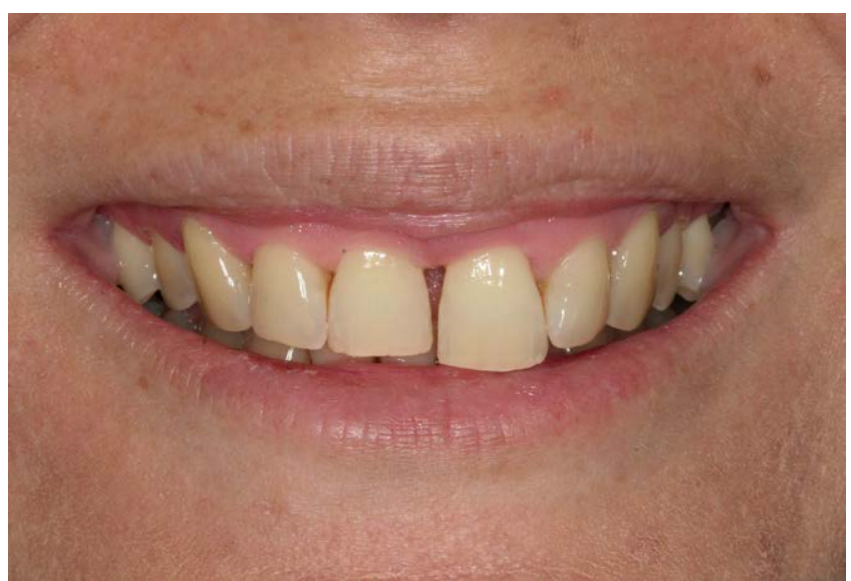

Abb. 1 Die 34-jährige Patientin konsultierte wegen der Zahnfehlstellung an Zahn 21 zunächst ihren Kieferorthopäden, der allerdings eine Parodontitis feststellte mit Sondierungstiefen von bis zu 10 Millimetern an Zahn 21. Daraufhin wurde die Patientin an die Sektion Parodontologie der Uniklinik Freiburg zur Parodontitistherapie überwiesen.

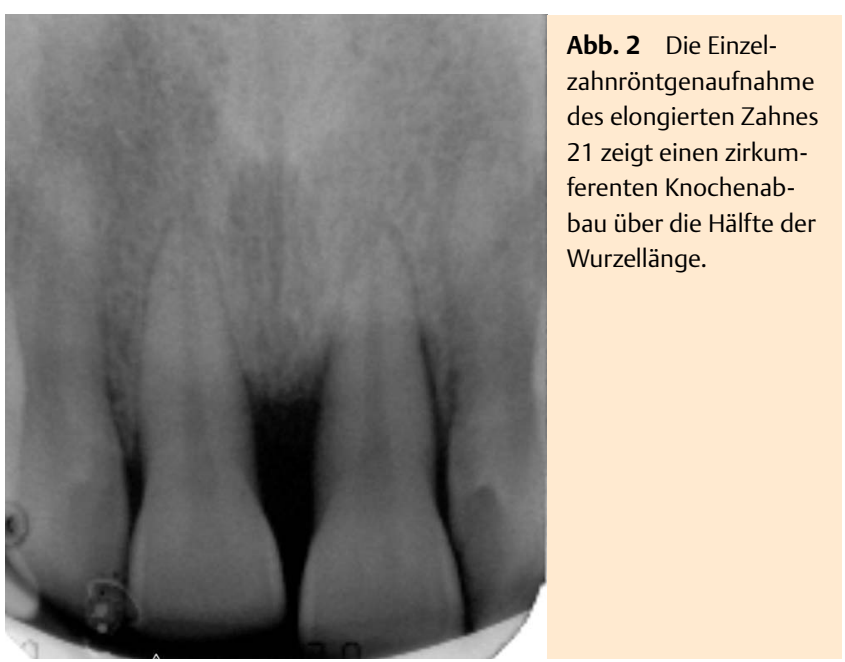


Im Rahmen der systematischen Parodontitistherapie ( $\bullet$ Abb. 4) wird darüber hinaus ein vollständiger Parodontalstatus mit Taschensondierungstiefen, Rezessionen, Bluten auf Sondieren, Furkationen und Lockerungsgraden, sowie ein Mundhygieneindex erhoben.

Als radiologische Basisdiagnostik bietet die Panoramaschichtaufnahme mit bedarfsmäßig zusätzlichen Einzelzahnfilmen eine gute Übersicht über die knöchernen Verhältnisse. In schweren Fällen und vor parodontalchirurgischen Maßnahmen bei Oberkiefermolaren kann die digitale Volumentomografie (DVT) von weiterem diagnostischem Nutzen sein [19].

\section{Therapie}

Die aktuellen Therapiekonzepte setzen im Wesentlichen auf die Kontrolle und Entfernung von bakteriellen Belägen und im weiteren auf die Modifikation bzw. Ausschaltung von Risikofaktoren

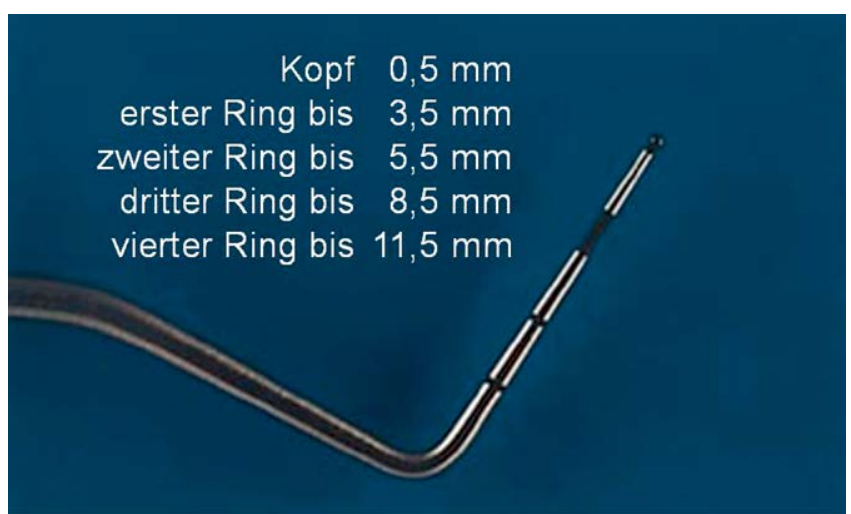

Abb. 3 Die WHO-Sonde als Erhebungsinstrument für den PSI (Parodontaler Screening Index). Das schwarze Band der Sonde kennzeichnet einen Sondierungstiefenbereich von 3,5 bis 5,5 Millimeter. und entzündungsförderlichen Faktoren des Wirtsorganismus wie z.B. Raucherentwöhnung oder Ernährungsberatung [20-23]. Im Rahmen der nicht chirurgischen Therapie erfolgen in mehreren Sitzungen sowohl eine Optimierung der häuslichen Mundhygiene, professionelle Zahnreinigungen, eine subgingivale Belagsentfernung bei Sondierungstiefen größer gleich $4 \mathrm{~mm}$ (Scaling und Wurzelglätten) und regelmäßige risikoorientierte Nachsorgebehandlungen ( $\bullet$ Abb. 3). Im Falle von persistierend erhöhten Sondierungstiefen über $6 \mathrm{~mm}$ können weitergehend parodontalchirurgische Maßnahmen indiziert sein.

In den letzten Jahren haben in diesem Bereich vor allem regenerative Maßnahmen mit Schmelz-Matrix-Proteinen vielversprechende Ergebnisse gezeigt [24]. In schweren Fällen, bei persistierenden Sondierungstiefen und bei aggressiven Parodontitiden bietet zudem die adjuvante Gabe von systemischen Antibiotika eine sinnvolle Behandlungsoption nach Abschluss des Scalings und Wurzelglättens [25]. Als wesentliches und langfristiges Element zur Erhaltung und parodontalen Gesunderhaltung der Zähne hat sich die regelmäßige Teilnahme an der parodontalen Nachsorge („Recall“) gezeigt [26]. Das parodontale Recall sollte dabei nach dem individuell berechneten Parodontitisrisiko entweder alle 3-4 Monate, jedes halbe Jahr oder einmal im Jahr erfolgen [27]. In die Risikoanalyse fließen dabei unter anderem die Zahl der erhöhten Sondierungstiefen größer gleich 5 Millimeter, das prozentuale Bluten auf Sondieren, der Knochenabbau im Verhältnis zum Alter, die Anzahl der verlorenen Zähne und das Vorliegen von systemischen Erkrankungen mit ein.

Bei der Therapie von Gingivarezessionen stehen vor allem die Aufklärung des Patienten und die Anamnese von Beschwerden, möglichen Ursachen und Erwartungen im Vordergrund. Grundlegend ist dabei eine Ausschaltung der ursächlichen Faktoren (wie z.B. aggressives Zähneputzen) und Umstellung der auf eine sanfte Reinigungsmethode mit geringerem Anpressdruck [28]. Die Art der mechanischen Reinigung (Handzahnbürste oder elektrische Zahnbürste) scheint in Bezug zur Rezessionsentstehung keinen Unterschied zu machen [29]. Indikationen für eine

\section{Code Kriterien}

0

Das schwarze Band der Sonde bleibt an der tiefsten Stelle des Sulkus eines Sextanten vollständig sichtbar. Zahnstein oder defekte Restaurationsränder sind nicht vorhanden.

1 Das schwarze Band der Sonde bleibt an der tiefsten Stelle des Sulkus eines Sextanten vollständig sichtbar. Zahnstein oder defekte Restaurationsränder sind nicht vorhanden. Nach (vorsichtigem) Sondieren tritt eine Blutung auf. Das schwarze Band der Sonde bleibt an der tiefsten Stelle des Sulkus eines Sextanten vollständig sichtbar. Es lassen sich Zahnstein oder defekte Restaurationsränder feststellen.

3 Das schwarze Band der Sonde bleibt an der tiefsten Stelle des Sulkus nur teilweise sichtbar.

4

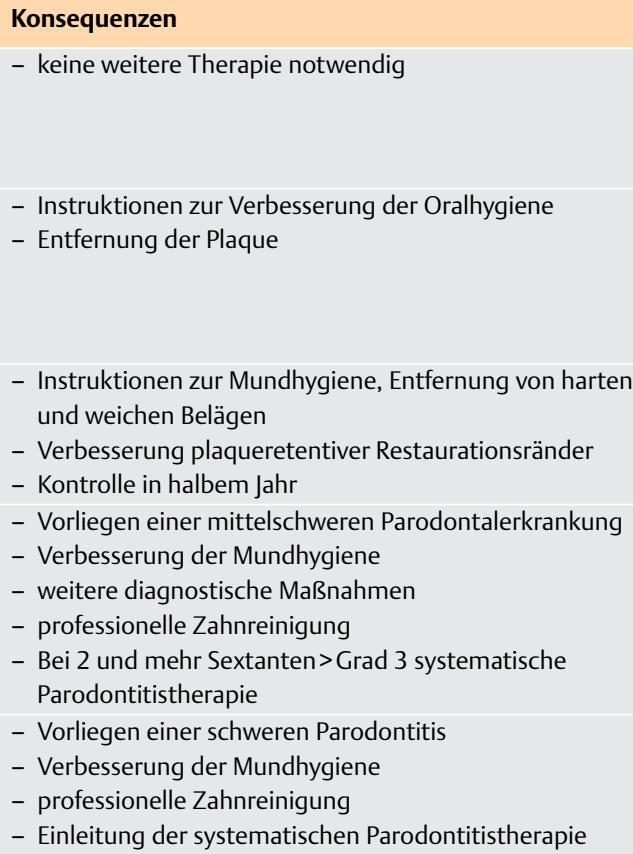

- Verbesserung plaqueretentiver Restaurationsränder

- Kontrolle in halbem Jahr

- Vorliegen einer mittelschweren Parodontalerkrankung

- Verbesserung der Mundhygiene

- weitere diagnostische Maßnahmen

- professionelle Zahnreinigung

- Bei 2 und mehr Sextanten>Grad 3 systematische Parodontitistherapie

- Vorliegen einer schweren Parodontitis

- Verbesserung der Mundhygiene

- professionelle Zahnreinigung

- Einleitung der systematischen Parodontitistherapie Sondierungstiefe gekennzeichnet, die größer als $5,5 \mathrm{~mm}$ ist.

Weitere klinische Besonderheiten (Furkationen, Gingivarezessionen ab 3,5 mm, Zahnbeweglichkeit, Mukogingivale Probleme) werden mit einem Sternchen $\left(^{*}\right)$ hinter dem Code versehen 
nicht-chirurgischen Parodontitistherapie

Ggf. Behandlung akuter Zustände
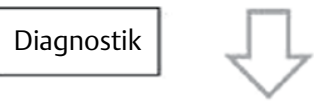

$$
\begin{aligned}
& \text { Kausale Therapie mit } \\
& \text { nicht-chirurgischen Maßnahmen } \\
& \text { - Information des Patienten } \\
& \text { - Mundhygieneinstruktion und -training, } \\
& \text { Motivation, Ernährungsberatung } \\
& \text { - Professionelle Zahnreinigung } \\
& \text { - Entfernung von Reizfaktoren } \\
& \text { - Subgingivales Scaling und } \\
& \text { Wurzelglätten } \\
& \text { - Ggf. antimikrobielle Therapie }
\end{aligned}
$$

Abb. 4 Schema der systematischen Parodontitistherapie.

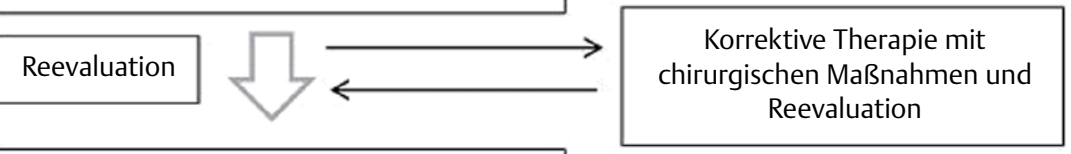

Erhaltungstherapie mit nicht-chirurgischen Maßnahmen

- Professionelle Zahnreinigung

- Remotivation

- Ggf. Nachbehandlung

plastische parodontalchirurgische Maßnahme können durch den ästhetischen Wunsch des Patienten, ein persistierendes mukogingivales Problem oder persistierende Dentinhypersensitivitäten, die nicht auf non-invasive therapeutische Maßnahmen (Fluoride, Versiegelungen) angesprochen haben, gestellt werden [30]. Die am häufigsten verwendeten Verfahren für die Rezessionsdeckung sind dabei der koronale Verschiebelappen, das Bindegewebstransplantat und Kombinationen beider Methoden [30]. Zudem besteht für diese Verfahren die beste verfügbare Evidenz [31].

\section{Interdisziplinäre Aspekte der Kieferorthopädie und Parodontologie \\ $\nabla$}

Eine der am häufigsten auftretenden Komplikationen bei kieferorthopädischer Therapie ist aus parodontologischer Sicht das Auftreten von gingivalen Rezessionen. Maßgebliche Faktoren für die Entwicklung von Rezessionen sind hierbei der Gingivaphänotyp (dünn/breit), das Vorhandensein von befestigter Gingiva, das Mundhygieneverhalten (zu wenig vs. zu viel), die gingivale Entzündung, der Abstand der knöchernen Strukturen zum Gingivalrand und eine kieferorthopädisch induzierte Knochendehiszenz [32,33]. In-vitro-Untersuchungen zeigen hierbei Vorteile von 3-dimensionalen Röntgenverfahren (DVT), um knöcherne Fenestrationen und dünne labiale Knochenverhältnisse vor Therapie einschätzen zu können [34]. Beim Vorliegen von Rezessionen sollte vor kieferorthopädischer Therapie immer eine parodontale Erkrankung ausgeschlossen werden. Unab- dingbar ist zudem eine Aufklärung der Patienten über die Entstehung von labialen Rezession bei Risikofaktoren wie einem dünnen Gingivaphänotypen oder einem zu hohen Anpressdruck beim Zähneputzen [32].

Eine andere Komplikation bei kieferorthopädischer Therapie kann die Entstehung von nicht entzündlichen Gingivawucherungen sein. Als Ursache hierfür werden Matrix-Metalloproteasen und entzündliche Prozesse im Parodontalligament durch die mechanische Krafteinwirkung, sowie allergische Reaktionen auf Nickel diskutiert [35]. In den meisten Fällen entwickeln sich die Gingivawucherungen nach Entbänderung zurück, in manchen Fällen müssen anschließende Gingivektomien vorgenommen werden [36].

Eine systematische Übersichtsarbeit zeigte eine moderate Evidenz, dass kieferorthopädische Maßnahmen zu einer Zunahme von parodontalpathogenen Keimen führen können [37]. Neben dem Faktor der erhöhten Plaqueretention durch die kieferorthopädischen Applikationen werden auch pro-inflammatorische Prozesse, die durch die forcierten Zahnbewegungen ausgelöst werden als förderlicher Faktor diskutiert [38]. Erfolgen Zahnbewegungen beim Vorherrschen von aktiven parodontalen Läsionen kann dies zu einem rapiden Knochenverlust beitragen [39]. Dementsprechend sollten gingivale entzündliche Veränderungen während der kieferorthopädischen Therapie immer als Warnhinweis verstanden werden und eine weitere parodontale Abklärung eingeleitet werden. Im Falle eines entzündungsfreien, aber reduzierten Parodonts nach Parodontaltherapie können im Gegensatz dazu sicher kieferorthopädische Maßnahmen durchgeführt werden [40]. Diese sollten dann allerdings regelmäßig auf parodontale Läsionen hin kontrolliert werden. 


\section{Zusammenfassung}

\section{$\nabla$}

Zusammenfassend lässt sich sagen, dass parodontale destruktive Prozesse durch eine klinische Inspektion und Diagnostik des Kieferorthopäden oder betreuenden Zahnarztes vor und während der kieferorthopädischen Therapie erkannt werden können. Präventiv stehen dabei vor allem die Erhebung des PSI, sowie eine Abklärung von parodontalen Risikofaktoren in der Anamnese vor kieferorthopädischer Therapie als Maßnahmen zur Verfügung. Während der Therapie sind intensivierte Mundhygienemaßnahmen zur Prävention der Gingivitis und Parodontitis von besonderer Bedeutung.

\section{Literatur}

1 Deutsche Gesellschaft für Parodontologie. Klassifikation der Parodontalerkrankungen. Berlin: Quintessenz; 2002

2 Löe H, Theilade E, Jensen SB. Experimental gingivitis in man. J Periodontol 1965; 36: 177-187

3 Brecx MC, Fröhlicher I, Gehr P et al. Stereological observations on long-term experimental gingivitis in man. J Clin Periodontol 1988; 15: 621-627

4 Socransky SS, Haffajee AD, Cugini MA et al. Microbial complexes in subgingival plaque. J Clin Periodontol 1998; 25: 134-144

5 Bartold PM, Van Dyke TE. Periodontitis: a host-mediated disruption of microbial homeostasis. Unlearning learned concepts. Periodontol 2000 2013; 62: 203-217

6 Marsh PD, Devine DA. How is the development of dental biofilms influenced by the host? J Clin Periodontol 2011; 38 (Suppl 11): 28-35

7 Hajishengallis G. Immunomicrobial pathogenesis of periodontitis: keystones, pathobionts, and host response. Trends Immunol 2014; 35: 3-11

8 Chapple ILC, Matthews JB. The role of reactive oxygen and antioxidant species in periodontal tissue destruction. Periodontol 2000. 2007; 43: $160-232$

9 Bullon P, Newman HN, Battino M. Obesity, diabetes mellitus, atherosclerosis and chronic periodontitis: a shared pathology via oxidative stress and mitochondrial dysfunction? Periodontol 2000 2014; 64: 139-153

10 Schaefer AS, Bochenek G, Manke T et al. Validation of reported genetic risk factors for periodontitis in a large-scale replication study. J Clin. Periodontol 2013; 40: 563-572

11 Michalowicz BS, Diehl SR, Gunsolley JC et al. Evidence of a substantial genetic basis for risk of adult periodontitis. J Periodontol 2000; 71: 1699-1707

12 Hoffmann T, John M, Kerschbaum $T$ et al. Vierte Deutsche Mundgesundheitsstudie (DMS IV). Köln. Mikrozensus: Fragen zur Gesundheit (2009a): Verteilung der Bevölkerung auf Body-Mass-Index-Gruppen in; 2006

13 Oh T-J, Eber R, Wang H-L. Periodontal diseases in the child and adolescent. Journal of Clinical Periodontology 2002; 29: 400-410

14 Albandar JM, Tinoco EMB. Global epidemiology of periodontal diseases in children and young persons. Periodontol 2000 2002; 29: 153-176

15 Grossi SG, Zambon JJ, Ho AW et al. Assessment of risk for periodontal disease. I. Risk indicators for attachment loss. J Periodontol 1994; 65: 260-267

16 Genco RJ, Borgnakke WS. Risk factors for periodontal disease. Periodontol 2000 2013; 62: 59-94

17 Gjermo P. CPITN as a basic periodontal examination in dental practice. Int Dent J 1994; 44: 547-552
18 Eickholz P. Glossar der Grundbegriffe für die Praxis. Parodontologische Diagnostik, Teil 5: PSI und Sondenparameter. Parodontologie 2010; 2: $177-187$

19 Woelber J, Fleiner J, Rau J et al. Accuracy and usefulness of CBCT in periodontology. A systematic review of the literature. Int J Periodontics Restorative Dent accepted

20 Dentino A, Lee S, Mailhot J et al. Principles of periodontology. Periodontol 2000 2013; 61: 16-53

21 Salvi G, Lindhe J, Lang N. Treatment Planning of Patients with Periodontal Diseases. In: Lang N, Lindhe J.editors. Clinical Periodontology and Implant Dentistry. $5^{\text {th }}$ ed. 2008: 654-674

22 Ramseier CA. Potential impact of subject-based risk factor control on periodontitis. J Clin Periodontol 2005; 32 (Suppl 6): 283-290

23 Chapple ILC. Potential mechanisms underpinning the nutritional modulation of periodontal inflammation. J Am Dent Assoc 2009; 140: 178-184

24 Koop R, Merheb J, Quirynen M. Periodontal regeneration with enamel matrix derivative in reconstructive periodontal therapy: a systematic review. J Periodontol 2012; 83: 707-720

25 Beikler T, Karch H, Flemming T. Adjuvante Antibiotika in der Parodontitistherapie. Wissenschaftliche Stellungnahme der DGZMK und der DGP. DZZ 2003; 58: 263-265

26 Eickholz P, Kaltschmitt J, Berbig J et al. Tooth loss after active periodontal therapy. 1: patient-related factors for risk, prognosis, and quality of outcome. J Clin Periodontol 2008; 35: 165-174

27 Lang NP, Tonetti MS. Periodontal risk assessment (PRA) for patients in supportive periodontal therapy (SPT). Oral Health Prev Dent 2003; 1: 7-16

28 Matas F, Sentís J, Mendieta C. Ten-year longitudinal study of gingival recession in dentists. J Clin Periodontol 2011; 38: 1091-1098

29 Dörfer CE, Staehle HJ, Wolff D. Three-year randomized study of manual and power toothbrush effects on pre-existing gingival recession. J Clin Periodontol 2016; 43: 512-519

30 Zucchelli G, Mounssif I. Periodontal plastic surgery. Periodontol 2000 2015; 68: 333-368

31 Chambrone L, Sukekava F, Araújo MG et al. Root coverage procedures for the treatment of localised recession-type defects. Cochrane Database Syst Rev 2009; CD007161

32 Merijohn GK. Management and prevention of gingival recession. Periodontol 2000 2016; 71: 228-242

33 Wennström JL. Mucogingival considerations in orthodontic treatment. Semin Orthod 1996; 2: 46-54

34 Patcas R, Müller L, Ullrich $O$ et al. Accuracy of cone-beam computed tomography at different resolutions assessed on the bony covering of the mandibular anterior teeth. Am J Orthod Dentofacial Orthop 2012; 141: 41-50

35 Gorbunkova A, Pagni G, Brizhak A et al. Impact of Orthodontic Treatment on Periodontal Tissues: A Narrative Review of Multidisciplinary Literature. International Journal of Dentistry 2016; e4723589

36 Kouraki E, Bissada NF, Palomo JM et al. Gingival enlargement and resolution during and after orthodontic treatment. N Y State Dent J 2005; 71: 34-37

37 de Freitas AOA, Marquezan M, Nojima $M$ et al. The influence of orthodontic fixed appliances on the oral microbiota: A systematic review. Dental Press J Orthod 2014; 19: 46-55

38 Zeng $M$, Kou X, Yang R et al. Orthodontic Force Induces Systemic Inflammatory Monocyte Responses. J Dent Res 2015; 94: 1295-1302

39 Kessler $M$. Interrelationships between orthodontics and periodontics. Am J Orthod 1976; 70: 154-172

40 Re S, Corrente G, Abundo R et al. Orthodontic treatment in periodontally compromised patients: 12-year report. Int J Periodontics Restorative Dent 2000; 20: 31-39 